\title{
Concept Map-based Aviation Competency Mapping and Training
}

\section{Dr. Yuetong Lin, Embry-Riddle Aeronautical University, Worldwide}

Yuetong Lin received the Ph.D. degree in Systems and Industrial Engineering from the University of Arizona, Tucson, in 2005. He was with Indiana State University from 2005 to 2016, where he became associate professor of Electronics and Computer Engineering Technology, and the program coordinator for Computer Engineering Technology. He joined Embry-Riddle Aeronautical University, Worldwide in September 2016 as an associate professor in the Department of Engineering and Technology.

\section{Dr. Ali Mehran Shahhosseini, Indiana State University}

A. Mehran Shahhosseini is an Associate Professor in the Department of Applied Engineering and Technology Management at Indiana State University. He has published over 45 articles in different journals and conference proceedings. He has served as an investigator for research projects sponsored by National Science Foundation, Ford Motor Company, and the US Army. Before working at Indiana State University, he was a faculty in the University of Louisville for 10 years. He also has over four years of industrial experience. He received his D.Eng. degree in Mechanical Engineering from Lamar University (USA) in 1999, M.Sc. in Materials Engineering from Isfahan University of Technology (Iran) in 1991, and B.Sc. in Metallurgical Engineering from Tehran University (Iran) in 1988. He is a member of ASEE, ASME, SAE, and ATMAE.

\section{Prof. Christian Janke, Embry Riddle Aeronautical University}

Christian Janke is an Assistant Professor at Embry Riddle Aeronautical Universities Worldwide Campus and the Associate Program Chair for the Bachelor of Science in Unmanned Systems Applications. He teaches at degree programs involving Unmanned Systems in all domains, including regulation, applications as well as Safety and Security.

He is a trainer for Human Factors \& Crew Resource Management, instructor for Safety Management and Risk Assessment in Aviation and Auditor for Quality Management. He has been in military service as a helicopter pilot and is a Research Fellow at the European Aviation Security Center. 


\title{
Concept map based aviation competency mapping and training
}

\begin{abstract}
Competency mapping is about identifying key skills required by an organization and/or a job so that they can be applied in important business operations such as job evaluation, training, and recruitment. For the organization, competency mapping serves two important purposes. On personal level, it analyzes individual's SWOT (Strengths, Weaknesses, Opportunities \& Threats) and helps him compare with the success criteria (i.e., behavioral standards of performance excellence) to improve his career growth. On corporate level, competency mapping reinforces corporate strategy, culture, and vision, and provides a common framework to improve the effectiveness of training and professional development programs.

In aviation industry, competency based workforce development has been implemented in the United Kingdom and Australia for pilot training, with the explicit objective of making employees globally competitive. Though the results are mixed, there is evidence that this approach appears to confer considerable advantages in specific areas for preparing pilots to operate aircraft. On the other hand, similar practice has not been established, or well designed for ground crew training. The safety department, for example, has a very urging demand to provide a common, organization-wide standard for different career levels that enable inspectors to smoothly adapt to new working environment and tasks.

One of the main issues with the current competency focused training is that it tends to be slow and expensive because of the need for extensive interviews and observations. These activities are very time-consuming for those outstanding performers and analysts. In addition, there is not a standard competency mapping process that works in all situations. Each company needs to have a unique model, which adds to the developing cost and time.

In this paper, we propose a competency mapping method based on conceptual maps for training in the aviation industry. The method builds on existing approaches and procedures, but incorporates new tools and analysis methods to assure the mapping to be concrete and comprehensive to provide effective guidance for ground crew training and skills appraisal.
\end{abstract}

\section{Introduction}

Operations in aviation, both military and civilian, require decision makings that are based on solid knowledge and more importantly, systematic thinking. Developing this skills set has been shown 
to be a challenge for both trainees and trainers. There are a few issues that can cause the training not meeting expectations, as pointed out by Air Force Training report to Congressional Committee $^{1}$. First, the step by step procedure laid out in the handbook or manual, which is the primary source for trainees for guidance, though offering direct instructions, is rigid and fails to provide a systematic view of the scenario and its solution to the trainee. Second, it has been recognized that the new generation of learners is more of the "visual learner" type. They absorb and understand domain knowledge better when they can have a pictorial representation of the system and solution.

The Federal Aviation Agency is the governing authority to regulate all aspects of civilian aviation in the United States. In many ways, it sets examples for other national aviation regulatory bodies in terms of operation management and workforce training. Over the past few years, the FAA has been eager, as evidenced by its numerous solicitations, to find better ways to help the agency determine the employees' skill levels so that training can be more targeted to improve their performances. For example, an Aviation Safety Inspector (ASI) working in Miami, FL may not have the same proficiency in a specific skill as an ASI working in Anchorage, AK (e.g., deicing procedures). Therefore when the ASI from Miami transfers to Anchorage, the agency needs a system to ensure the gap in proficiency is identified and the correct courses are scheduled to address it. Examples like this show the urgency of developing consistent methods to answer the question: What best practices and methods can be applied in order to map all the position descriptions in AFS, to the competencies, skills, behaviors, and proficiencies needed so that appropriate employee development can be conducted in a timely manner.

There have been many attempts taken to address these critical training demands. Several programs have been developed that share common features such as computer-based tools and taking into consideration of "human factors" in the aviation maintenance operations. Liang et al. ${ }^{2}$ propose an on-line maintenance assistance platform (on-line MAP) for technicians to perform maintenance tasks to increase aviation maintenance and inspection safety. The platform uses graphic information aids to improve teams' risk cognition, situation awareness, technicians' performance and their job satisfaction comparing with the current work-card instruction. Sadasivan and Gramopadhye ${ }^{3}$ develop a computer-based inspection training tool, General Aviation Inspection Training System, that uses a multi-media presentational approach with interaction opportunities between the user and the computer for training aircraft maintenance technicians' inspection skills. The authors also point out the importance of standardizing the inspection training process, developing guidelines-based human factors research through the identification and implementation of intervention strategies, and providing an industry-wide benchmark for inspection training. Das et al. ${ }^{4}$ present an interactive tool called ASK-the-Expert and its application in the aviation safety applications that allows subject matter experts to review instances from a data set and provide labels within a single framework. Dalkilic ${ }^{5}$ introduces a training model developed in accordance with EASA Part-66 requirements and delivered by e-learning methods, and shows its advantages over traditional face to face training models. However, these programs lack sufficient support for the skills mapping, which is a high priority in FAA's training requirements. And this is where competency mapping paradigm can potentially excel.

Competency mapping requires a well defined competency model, which is a valid, observable, 
and measurable list of the knowledge, skills, and attributes demonstrated through behavior that results in outstanding performance in a particular work context ${ }^{6}$. However, traditional approaches for modeling have not fully leveraged the latest technology and analysis techniques to generate and synthesize data. For application of competency mapping in aviation training to be efficient, we need to develop faster and accurate methods for both modeling and analysis.

To achieve this goal, we turn to basic cognitive and instructional theory. Studies in cognitive psychology have shown that internal representations of knowledge are structured in a web where the creation of more connections usually leads to better understanding. When individuals need to retrieve pertinent information to perform domain-specific tasks, researchers have found that increased structural integration of mental models lead to increases in the efficiencies of knowledge that can be used to solve a given problem. While there is a high-level of homogeneity among field experts' models, those models vary significantly from models produced by novices. This indicates that models can differentiate disparate levels of comprehension. Therefore, by externalizing internal understandings, we can show one's thought process to solve problems and leverage the discrepancy between expert and novice conceptualizations to achieve better learning outcomes. Extending this analysis to competency mapping, we believe current methods fail to represent associative networks of knowledge, or to exploit the idea that knowledge is stored in a network format where concepts are connected to each other. It is readily conceivable that a better competency mapping should include graphical schematics of experts' perceptions of the task's components, of their cognitive organization of routine elements, and of the relationships between these elements. And with the advancement of technology, this process should be formulated as a collaborative activity between humans and computers where computers help to elicit mental models and analyze results.

This is the foundation for the well-established conceptual map learning paradigm, which has seen wide adoption in education and training. Concept maps are at the core of our proposed competency mapping model for aviation training, whose underlying mechanism requires both trainers and trainees to communicate through visual representation of the task and proposed solution.

\section{Concept Map based Training}

Conceptual mapping is a well-recognized approach that uses both content knowledge and process knowledge to prompt users to create visual maps of a diagnostic strategy to identify technical problems in complex technical systems ${ }^{7}$. Although often time a complicated and time-consuming knowledge acquisition process, conceptual mapping is nonetheless a good tool to portray knowledge structure and to diagnose learner's misconception in learning. A comprehensive study of applications of concept maps in higher education is provided by Pia et al. ${ }^{8}$. Morsi et al. ${ }^{9}$ present a review of concept mapping applications in engineering disciplines in general and in Electrical and Computer Engineering in specific.

Between 2010 and 2013, our team received a National Science Foundation TUES grant to help develop software to advance diagnostic skills training for the undergraduate technology and engineering students. We developed the training modules using concept maps that required 
students to draw visual maps of a troubleshooting strategy. As illustrated in Figure 1, these student maps would be compared with maps created by subject experts on the same task to gauge students' mastery of the desired diagnostic skills and areas of improvement.

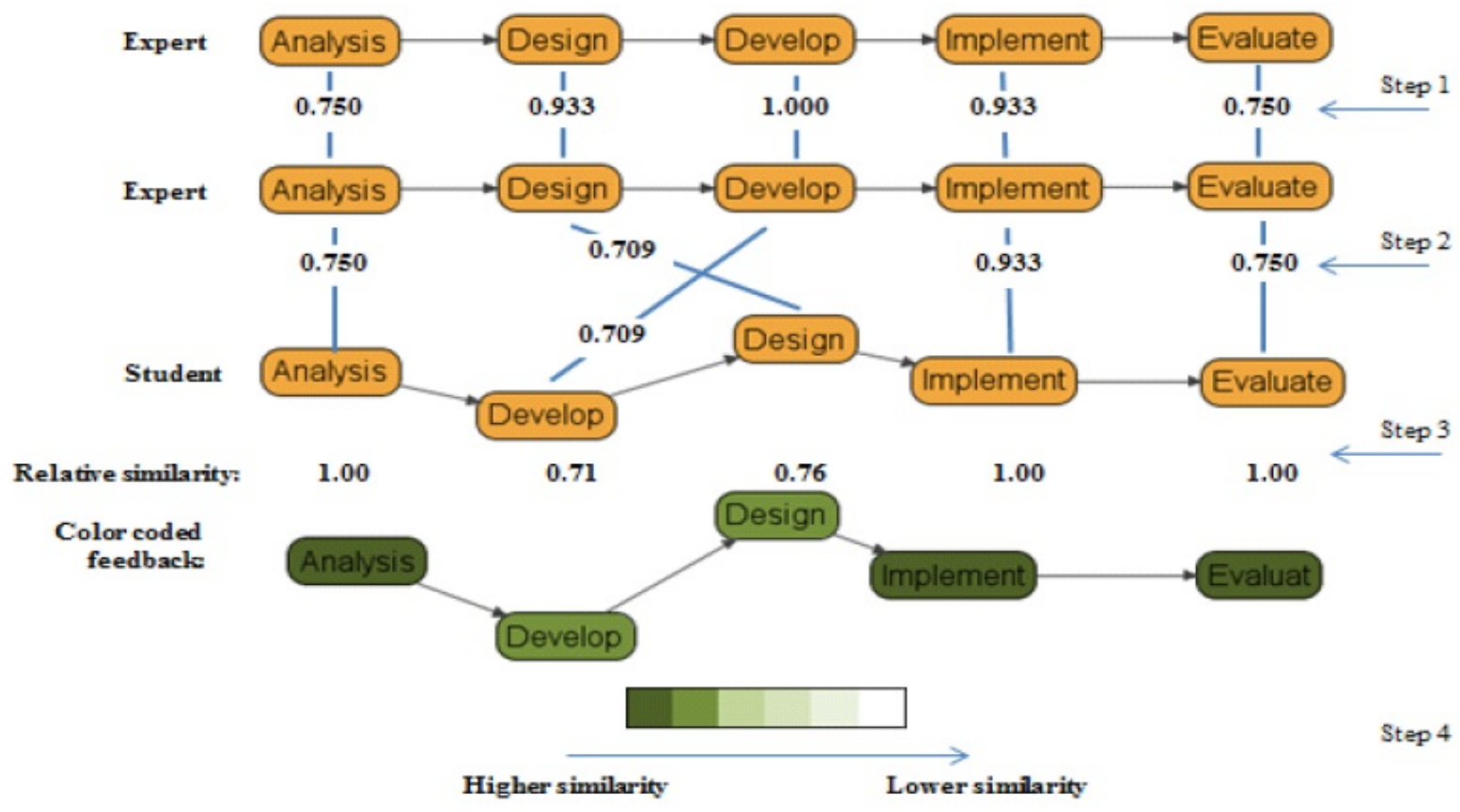

Figure 1: The process of comparison for process maps

One of the biggest challenges for concept maps based methods is how to evaluate and compare maps. Not only we need to assure that the mechanism recognizes both structural and semantic differences in the maps, we also hope to ditch the labor intensive and time consuming manual comparison and let computers dissect the results without human involvement. There are several noteworthy studies on this subject. Limongelli et al. ${ }^{10}$ propose seven measures of similarity among concept maps dealing with both structural and didactic aspects of the maps. A weighted concept is proposed by Chang et al. ${ }^{11}$ where propositions are given a weight value from 0 to 1 , based on the closeness index and weighted value of each node, a similarity index is calculated for each node. Gao et al. ${ }^{12}$ present an approach of string comparison with the meaning of the words-semantic similarity. Melnik et al. ${ }^{13}$ presented a method called Similarity Flooding Algorithm (SFA). Our method of comparing concept maps is based on combining the weighting mechanism, SFA, and semantic similarity of two strings. The detail introduction of the method is included in the paper by Shahhosseini et al. ${ }^{14}$ and shown in Figure 2. In Figure 2, we adopt SFA program (Open source Java code developed by Melnik et al.) to match the nodes based on their relationships. The SFA represents two input maps semantically in code first, then creates an initial map for the product of each node in both maps, calculates their similarities based on the links, and finally generates a list of best paired nodes according to the similarity of each pair. During the comparison, WordNet ${ }^{\circledR}$ is used to measure the content of nodes.

The first step in comparing process is to generate a reference similarity that is calculated based on the result of comparing an expert's map with the expert's map itself $\left(s_{\text {aee }}\right)$. The reference 


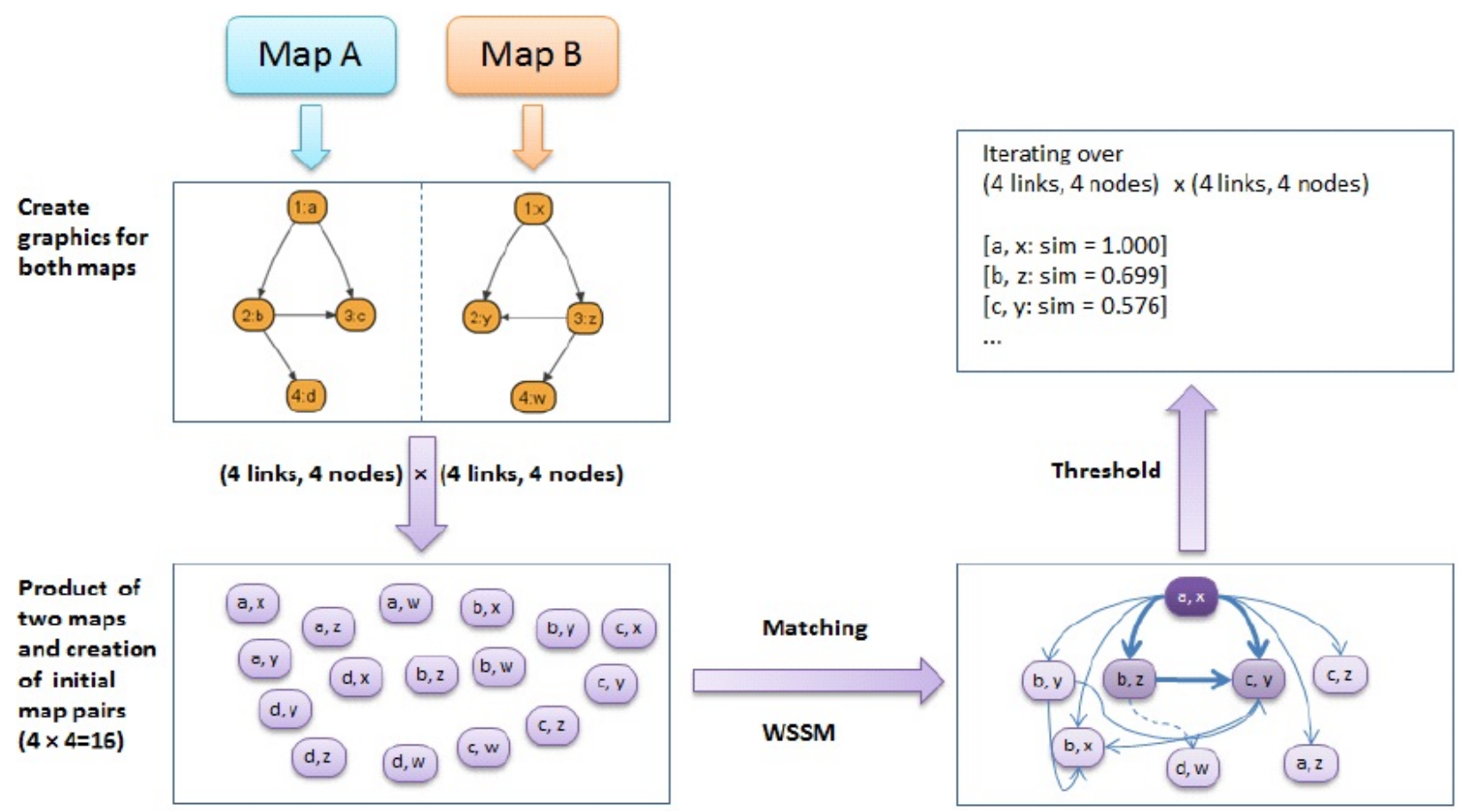

Figure 2: SFA Process

similarity includes absolute similarity for each paired nodes, according to their links and content. The second step is to compare a student's map with the expert's map $\left(s_{\text {ase }}\right)$. The comparison result includes absolute similarity for each node, which is considered as a matched pair with one node in the expert's map. The third step is to calculate the relative similarity $\left(s_{\text {rse }}\right)$ for each paired nodes by the percentage of $s_{a e e}$ and $s_{a s e}$. And, the overall similarity of the map is calculated based on the relative similarity of each pair. In this step, weighting $(w)$ of each pair can be considered. Important nodes can have more weighting in overall similarity.

Later, the team was invited to participate in NSF's I-Corps. The objective was to learn how to identify industrial partners so that the diagnostic training programs can be adopted or adapted to tackle practical problems. During the month-long customer interactions, the team had interviewed over 100 potential clients, the majority of whom were engineers, managers, and directors of operations in heavy industry like energy, manufacturing, or health care sector. The concept map based approached was well received by our interviewees and they believed the idea has the potential to help the companies, especially small business to standardize their training process and retain field experiences, often called "tribal knowledge" from their veteran employees.

To demonstrate how concept map based training module would work aviation training, we reference the following case study about a diagnostic task in electrical power generation and transmission, courtesy of Duke Energy. Figure 3 shows the concept map developed by an expert that details the diagnosis thought process in a systematic way. Students are provided with the performance data of the past thirty-six hours on a boiler, a wind turbine, and a transmission line, and are required to develop their own visual maps to choreograph how the maintenance team could use these data to pinpoint the problem. 


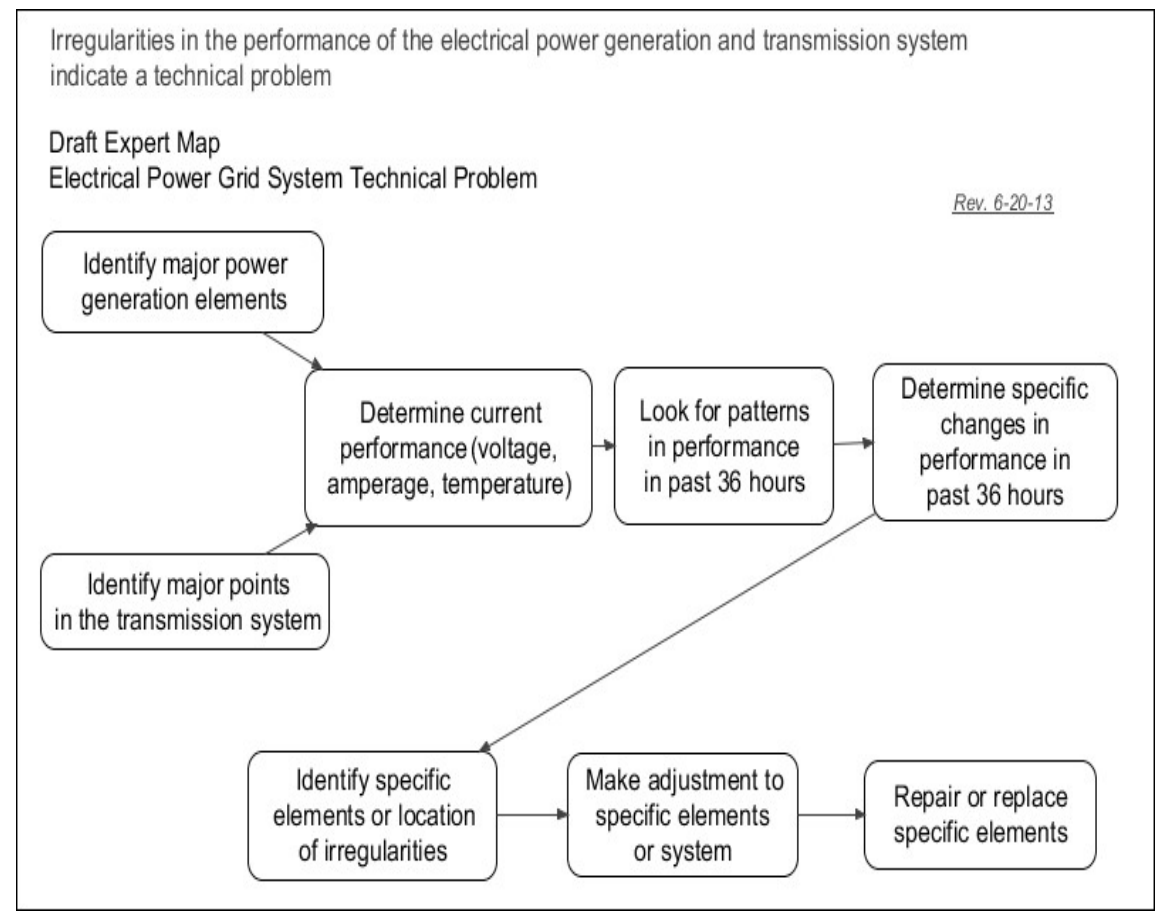

Figure 3: Expert's Map for Power Grid Problem in the Training Program

In the first attempt, the student's map has an $18 \%$ overall similarity and $38 \%$ "considerable" node similarities to the expert's map (range from $29 \%$ to $70 \%$ ), as summarized in Figure 4 . The student's map is shown in 5, where shade of the nodes indicates their semantic resemblance to their counterparts in the expert's map. There are three nodes with green colors. One is darker and the other two are lighter. The darker the color of the node is, the more similarity the node has. This case is from the control group, which means that the student only can see the comparison feedback before developing the second map.

After the student reviews feedback provided by the comparison program, he creates a new map shown in Figure 6. For this student, Figure 7 indicates that the second map sees an increase of $14 \%$ in overall similarity (from $18 \%$ to $32 \%$ ), and $25 \%$ in the matched nodes (38\% to 63\%). This increase is due to the fact that the new map uses a linear structure to replace the branch layout in the first try, which makes it more inline with the expert's map.

\section{Framework for Concept Map based Aviation Competency Mapping}

Many literatures have pointed out that a significant percentage of air transport accidents is caused by human maintenance errors, or at least the errors start the accident-chain ${ }^{15,16}$. And the severity of the issue is only getting worse due to the complexity of contemporary aircraft. It is clear that proper training of aircraft maintenance and inspection personnel will avoid failures, reduce maintenance related accidents, improve safety and reliability in aviation.

According to Walter ${ }^{15}$, more than $90 \%$ of the critical skills that an aviation maintenance or 


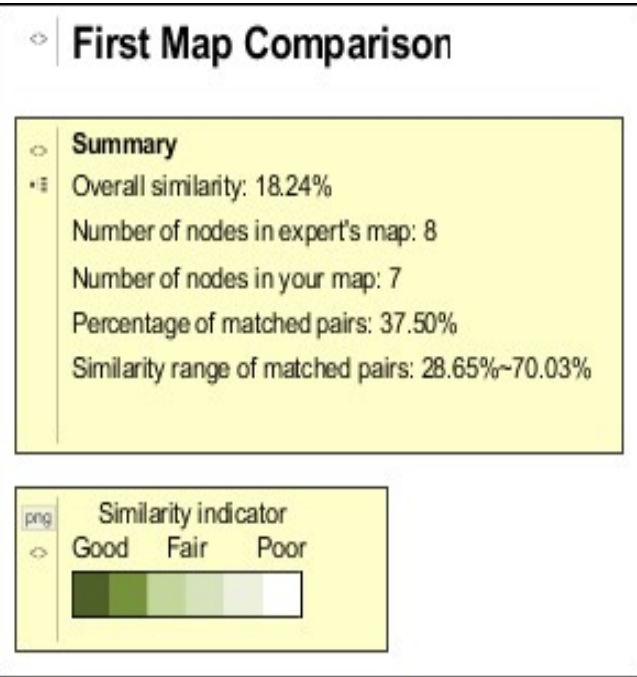

Figure 4: Power Grid Maps Comparison Summary: First Attempt

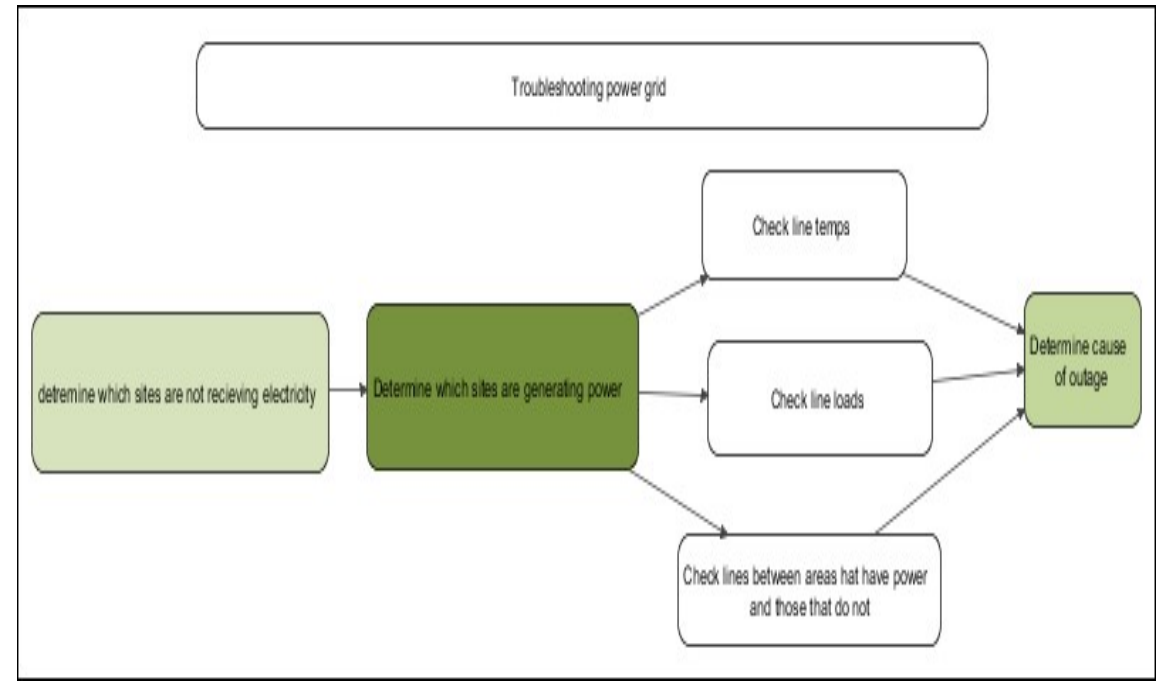

Figure 5: Student's Map for Power Grid Problem: First Attempt 


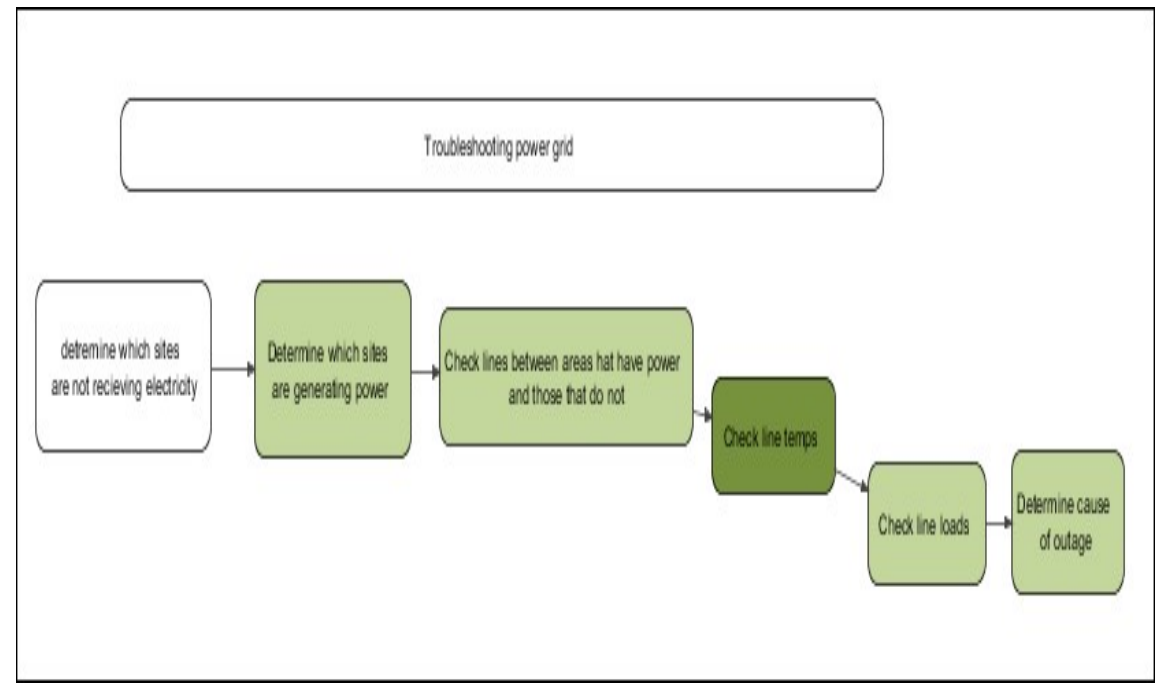

Figure 6: Student's Map for Power Grid Problem: Second Attempt

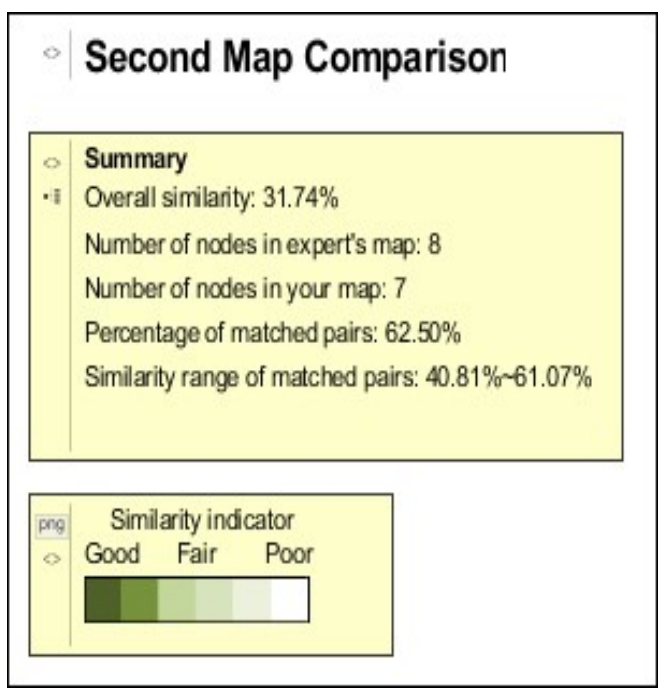

Figure 7: Power Grid Maps Comparison Summary: Second Attempt 
inspection technician uses are acquired through very unstructured on-the-job training. The conundrum in aviation training is somewhat similar to training new employees in heavy industry for system diagnosis and troubleshooting. Based on our I-Corps feedback, we believe the diagnostic skills training modules that we have tested in previous section can be the foundation for a structured and efficient aviation training program as well.

Recognizing the heterogeneous nature of job skills for aviation maintenance and inspection evidenced in the previous example, we realize one-size-fit-all type of framework is not practical. Therefore, we will follow a multiple-job approach, which offers the dual advantages of a common conceptual framework and customization for individual jobs.

The framework of the training will include $a$ ) a database comprising of the identified competencies; $b$ ) a presentation layer to conceptualize the specific skill for the user, and $c$ ) a mapping engine to link up the user-context to the appropriate competency.

\section{Conclusions}

In this paper, we have proposed a concept map based paradigm to help aviation industry conduct competency mapping and training. This is still a work in progress because the framework has not been validated by any practical training scenarios. Our next step will be to collaborate with aviation professionals to build concept maps for their key maintenance and inspection tasks. These maps will serve two purposes. First, they can be used to link skills with jobs, which offers a viable solution to competency mapping that is highly desired by FAA and many commercial airlines. Second, these maps help archive field expertise from long time staffs and technicians and provide visual guidance for new employees' training needs. Based on the experience of applying this framework to power and manufacturing diagnosis training, we are confident it will produce the needed outcomes for similar jobs in aviation industry.

\section{References}

[1] United States Government Accountability Office. Air force training: Further analysis and planning needed to improve effectiveness, 9 2016. URL https://www. gao.gov/assets/680/679849.pdf. Report to Congressional Committees.

[2] Guo-Feng Liang, Jhih-Tsong Lin, Sheue-Ling Hwang, Eric Min yang Wang, and Patrick Patterson. Preventing human errors in aviation maintenance using an on-line maintenance assistance platform. International Journal of Industrial Ergonomics, 40(3):356 - 367, 2010.

[3] Sajay Sadasivan and Anand K. Gramopadhye. Technology to support inspection training in the general aviation industry: Specification and design. International Journal of Industrial Ergonomics, 39(4):608 - 620, 2009. Special issue: Felicitating Colin G. Drury. 
[4] Kamalika Das, Ilya Avrekh, Bryan Matthews, Manali Sharma, and Nikunj Oza. Ask-the-expert: Active learning based knowledge discovery using the expert. volume 10536 LNAI, pages 395 - 399, Skopje, Macedonia, 2017.

[5] Serdar Dalkilic. Improving aircraft safety and reliability by aircraft maintenance technician training. Engineering Failure Analysis, 82:687 - 694, 2017.

[6] Srinath Perera, John Pearson, Damilola Ekundayo, and Lei Zhou. Professional, academic and industrial development needs: a competency mapping and expert opinion review. International Journal of Strategic Property Management, 17(2):143-160, 2013.

[7] J.D. Novak. Learning, Creating, and Using Knowledge: Concept Maps(tm) As Facilitative Tools in Schools and Corporations. Taylor \& Francis, 1998.

[8] Amparo Bes Pia, Encarna Blasco-Tamarit, and Maria Jose Munoz-Portero. Different applications of concept maps in higher education. Journal of Industrial Engineering and Management, 4(1):81 - 102, 2011. ISSN 20138423.

[9] Rasha Morsi, Wael Ibrahim, and Frances Williams. Concept maps: Development and validation of engineering curricula. In Proceedings - Frontiers in Education Conference, FIE, pages T3H18 - T3H23, Milwaukee, WI, United states, 2007.

[10] Carla Limongelli, Matteo Lombardi, Alessandro Marani, Filippo Sciarrone, and Marco Temperini. Concept maps similarity measures for educational applications. In Lecture Notes in Computer Science (including subseries Lecture Notes in Artificial Intelligence and Lecture Notes in Bioinformatics), volume 9684, pages 361 - 367. Springer, Zagreb, Croatia, 2016.

[11] Kuo-En Chang, Yao-Ting Sung, Rey-Bin Chang, and Shui-Cheng Lin. A new assessment for computer-based concept mapping. Educational Technology \& Society, 8(3):138-148, 2005.

[12] Jian-Bo Gao, Bao-Wen Zhang, and Xiao-Hua Chen. A WordNet-based semantic similarity measurement combining edge-counting and information content theory. Engineering Applications of Artificial Intelligence, 39:80-88, 2015.

[13] Sergey Melnik, Hector Garcia-Molina, and Erhard Rahm. Similarity flooding: A versatile graph matching algorithm and its application to schema matching. In Data Engineering, 2002. Proceedings. 18th International Conference on, pages 117-128. IEEE, 2002.

[14] A. Mehran Shahhosseini, Haisong Ye, George Maughan, and Tad Foster. Implementation of similarity flooding algorithm to solve engineering problems using diagnostic skills training technique. In ASME 2014 International Mechanical Engineering Congress and Exposition, pages V005T05A023-V005T05A023. American Society of Mechanical Engineers, 2014.

[15] Diane Walter. Competency-based on-the-job training for aviation maintenance and inspection - a human factors approach. International Journal of Industrial Ergonomics, 26(2):249 - 259, 2000.

[16] O. Usanmaz. Training of the maintenance personnel to prevent failures in aircraft systems. Engineering Failure Analysis, 18(7):1683 - 1688, 2011. 